\title{
Abstracts of papers presented at the Mammalian Genetics Group meeting, held in the Linnean Society Rooms, Piccadilly, London on 29 and 30 November 1984
}

\section{Lysosomal mutations affect response to anaesthesia}

\author{
By FAIZAH AHMED ANd JOHN G. M. SHIRE \\ Department of Biology, University of Essex, Colchester CO4 3SQ
}

Mutations at several loci affect both melanosomes and lysosomes. They include beige $(b g)$ and reduced pigmentation $(r p)$ (Genet. Res. 37, 95, 1981) in the house mouse. We have investigated the pleiotropic effects of these mutations by comparing homozygotes with heterozygous litter-mates of the same sex. Bleeding time in $r p r p$ mice is markedly increased, as it is in beige mice (Proc. Soc. exp. Biol. Med. 151, 32, 1976), implying a significant defect in serotonin metabolism in the mutant platelets. The duration of anaesthesia, measured by the length of time for the righting reflex to return, was examined for both mutations using two different anaesthetics. These were pentobarbital and amylene hydrate. Both homozygotes had significantly longer sleeping times than their heterozygous litter mates. The effects were more pronounced in beige mice, in females and with pentobarbital.

\section{Generating mouse mutants to dissect complex mammalian systems: from the $t$-region to phenyketonuria}

\section{By VERNON C. BODE, MONICA JUSTICE AND JAMES D. MCDONALD \\ Division of Biology, Kansas State University, Manhattan, Kansas 66506 U.S.A.}

Mouse mutants that are useful for dissecting a particular disease, a developmental pathway or a genetic system can be obtained by ENU mutagenesis of spermatogonia followed by progeny screening. This has been demonstrated in our laboratory by the isolation of new mutant alleles for the following genes on chromosome 17: tail interaction $(t$-int), quaking $(q k)$ and tufted $(t f)$ in normal chromatin, and brackyury $(T), q k$ and $t f$ in chromatin of $t^{\mathrm{w} 5}$ haplotype. The new $q k^{\mathrm{k}}$ and $q k^{\mathrm{kt}}$ alleles exhibit some properties which are different from the existing mutant. The new mutations in the $t^{\mathrm{w} 5}$ haplotype will be used to determine the extent of gene rearrangement in $t$-chromatin and should serve as landmarks in sequence studies at the DNA level. Numerous visible dominant mutations were observed for alleles on other chromosomes including the $X$. In these experiments we screened the first generation offspring of mutagenized males mated to females carrying mutations which permitted expression of the recessive mutant phenotype. This technique is not 
suitable when the mutations are recessive and the genes have no known mutant alleles, or when the new allele cannot be distinguished from the one used to detect its occurrence. A three generation breeding scheme is described which generates homozygous mutant animals. The isolation of a mutant with hereditary hyperphenylalanemia documents the validity and the potential power of this method. The properties of this mutant will be discussed. We believe that these experiments document the general utility of this method for obtaining specific mutants.

\title{
The progenitor concept in ontogenesis: Dendogram of 19 tissues derived from PGK-1B/-1A mosaic compositions in female mice.
}

\author{
BY TH BÜCHER, M. DÜNNWALD, I. M. LINKE AND H. M. RABES, \\ Institutes of Physiological Chemistry and Pathology, University of Munich, \\ Goethestr. 33, D8000 München 2
}

The progenitor cells concept, defined by MacLaren and extended by Falconer and Avery, is a statistical one. Ontogenetic sampling events of allocation leading to the progenitors of foetal cell lines are inferred from between-and within-individual variations in resultant organs. Thus, from the two possible approaches to the ontogenetic tree, starting forwards from the egg and working backwards from the finished product, the progenitor concept belongs to the latter. Compositions of genetically marked mosaics are measured and correlated on the basis of statistical models. Recently, correlations in the mosaic composition of the liver lobes of heterozygous female mice were studied (Gen. Res. Camb. 41, 31 1983). Allozymes of $X$-linked phosphoglycerate kinase served as markers. Our findings suggested a separation of the seven liver lobes into at least two different developmental populations. Hence, entering upon the mouse liver as an ontogenetic entity might be misleading. In principle, applications of the progenitor concept must be preceded by an experimental delimitation of the progenitor dependent cell communities. Preliminary investigations along those lines will be communicated. Dendrograms computed by the means of the CLUSTAN program with a data matrix of allozyme compositions of 19 tissues within 10 females, heterozygous at Pgk-1 but homozygous at the $\mathrm{Xce}$-locus $(X c e-c / X c e-c)$, will be presented.

\section{Chromosome 11 parental source effect upon foetal growth in mice}

\author{
By B. M. CATTANACH AND M. KIRK \\ MRC Radiobiology Unit, Harwell, Didcot, Oxon OX11 ORD, U.K.
}

Intercrosses between heterozygotes for Robertsonian translocations which have high rates of non-disjunction allows the production of chromosomally balanced young which have both members of an homologous pair of chromosomes derived from one parent. Such young are viable and apparently normal when either 
maternally or paternally disomic for chromosomes, $1,4,5,9,14$ or 15 , or maternally disomic for chromosomes 6 and 8. Maternal disomy 11, however, results in young which are only about one-half normal size at birth, while paternal disomic 11 young are about one and a half times bigger than their normal sibs. Both sexes are affected, viabilities are normal and growth rates parallel those of normal mice to maturity to give small and large adults, respectively. Both small and large mice are fertile and their young are of normal size. Intercrosses between $T(2 ; 11) 30 H$ heterozygotes are demonstrating the same phenomenon and suggest that the effect is associated with the proximal region of chromosome 11 . This carries the locus for $d f$, a growth hormone mutant. Since foetal growth is not dependent upon the foetus's own growth hormone it is suspected that (1) the size differences are secondary consequences of differences in placental growth and development, this perhaps hormonally controlled, and (2) the parental origin effect derives from an imprinting process expressed in extra-embryonic membranes whereby the paternally derived chromosome 11 (s) is (are) preferentially expressed.

\title{
Steroid sulphatase and the spread of $\mathrm{X}$-inactivation in man
}

\author{
BY A. J. M. CROCKER \\ Department of Medical Genetics, Churchill Hospital, Headington, Oxford, \\ OX3 $7 L J$
}

Normal female levels of STS have been found in a female of the karyotype $45 X$, ter rea $(X ; 15)(p 22 ; 26)$. This indicates that the STS locus has not been deleted. It is difficult therefore to explain why 97/100 lymphocytes show inactivation of the normal $X$, since $X$-inactivation would not normally spread beyond the STS locus. These results will be discussed in relation to the control of $X$-inactivation spreading. Results of studies on STS levels in the Djungarian Hamster and Guinea Pig will also be presented, and the status of $X$-inactivation of STS in mammals generally will be discussed.

\section{Unstable genes in mammals}

\author{
By M. S. DEOL AND G. M. TRUSLOVE \\ Department of Genetics and Biometry, University College London, Gower Street, \\ London WC1E 6BT
}

There are certain genes in the mouse that appear to have an extremely high rate of mutation in somatic cells. They all affect pigmentation, and the result is inherited mosaicism of coat colour. One of these genes may be different from the others: mosaic animals may produce self-coloured offspring, which breed true, at least for two generations. Superficially, the effects of these genes resemble Position Effect Variegation (PEV) in Drosophila: for instance, different tissues are affected 
to different extents, and there can be clear regional variation within the same structure. They can all be explained on the same basis as PEV : a chromosomal rearrangement, perhaps too small to be cytologically detectable, places the gene next to a heterochromatic region, with the result that in some cells the inactivating effect of the heterochromatin spreads into it. The possibility that transposable elements may be involved cannot be ruled out, for they can also suppress gene activity. These genes were evidently detected because they affect an easily classifiable trait for which a large number of animals are routinely examined. When the literature about comparable traits in man was searched, it was found that six families with inherited mosaicism of the ABO blood group system had been reported, and they could all be explained on the same basis.

\title{
Activity levels of the maternally and paternally derived phospho-glycerate kinase allozymes PGK-1B and PGK-1A in early mouse embryos homozygous at the Xce locus
}

\author{
By W. K. G. KRIETSCH, R. FUNDELE, M. FEHLAU AND TH BÜCHER
}

Institut für physiologische Chemie der Universität München, Goethestrasse 33, D8000 München 2, Germany

Activity levels of phosphoglycerate kinase allozymes in the unfertilized eggs of heterozygous mice and in early embryonal stages were recently measured in London and in Munich. Asexpected, an expression of both maternal $X$-chromosomes was observed. Unexpectedly, however, skewed patterns were found, the mean activity of PGK-1B exceeding the one of PGK-1A by the factor of nearly two. Non-random expression of $X$-chromosomes carrying the Pgk-1 alleles is also a common phenomenon in somatic phenotypic mosaicism of the $\mathrm{C} 3 \mathrm{H}$ mouse. But here just the inverted proportion of PGK-1B and PGK-1A expressing cells occurs. The cause of non-random lyonization of $X$-linked markers is thought to reside in accompanying heterozygosity at the $X c e$ locus. In order to test the possibility that the indicated imbalances are caused by such heterozygosity at the Xce-locus or by another $c i s$-acting factor in the vicinity of the structural $P g k$-1 gene, feral males were caught at the same site where the Pgk-1 ${ }^{a}$ allele was discovered (J. T. Neilsen, unpublished). Their $X$-chromosome carrying not only $P g k-1^{b}$ but, probably, also a genetic neighbourhood similar to the one of $P g k-1^{a}$ were introduced into the $\mathrm{C} 3 \mathrm{H} / \mathrm{Aa}$ strain. After nine generations of backcrossing, $P g k-1^{b} /-1^{a}$ heterozygote offspring exhibits in fact not only balanced phenotypic mosaicism but also well balanced activity patterns in the eggs and in the embryos, at decreasing absolute levels, up to early blastocysts. With the transition to the late blastocyst the individual allozyme pattern changes, however, to unimodality of rapidly increasing PGK activities. Of 33 embryos which were analysed at the late blastocyst stage 13 exhibited 80-100 per cent PGK-1B and 20 exhibited 80-100\% PGK-1A. In confirmation of earlier results any activation of foetal Pgk-1 genes of paternal derivation must be excluded. This observation can be correlated to the exclusive 
expression of maternally derived $X$-chromosomes in the trophectoderm and primary endodermal lineages of heterozygote embryos. At the stage of allocation of these lineages during the transition from the early to the late blastocyst foetal chromosomes might be still inactive, and the biogenetic processes supporting the development might mainly depend upon the activities furnished by the mother during oogenesis. Consequently, in addition to maternal imprinting of the $X$ chromosomes, also maternally derived imprinting of the blastocysts plasma membranes might be considered.

\title{
Do distorter genes cause male sterility of $t$-haplotypes?
}

\author{
By M. F. LYON \\ MRC Radiobiology Unit, Harwell, Didcot, Oxon OX11 ORD, U.K.
}

Distortion of transmission ratio in male mice heterozygous for $t$-haplotypes is due to the action of 3 or more distorter genes, $T c d$, acting on a responder, $T c r$, so as to cause the chromosome carrying the responder to be transmitted at a high frequency. Male sterility in $t$-haplotypes also depends on a number of genes. The three so far identified are located in the same regions of the $t$-complex as the three distorters, and it is suggested that they are identical with them. Heterozygosity for distorters is sufficient to produce abnormal transmission, but homozygosity at one or more distortion loci is required for male sterility. An exception to this rule was provided by a combination of distorters $T c d-1$ and $T c d-2$, which caused markedly reduced fertility when both genes were heterozygous, and wild-type alleles were present at the responder locus. Animals heterozygous for the $t$ form of the responder, as well as for $T c d-1$ and $T c d-2$, were normally fertile. The suggested interpretation is that the distorters impair the functioning of sperm through an action on the responder. The wild-type allele of $T c r$ is more sensitive than the $t$-form, leading to distorted transmission. However, when distorters are homozygous the effect is severe enough to affect the $t$-form of $T c r$, as well as wild-type, resulting in sterility.

\section{Synaptonemal complex analysis in spermatocytes and oocytes of $\operatorname{Ts}\left(5^{12}\right) 31 H$ mice}

\author{
By SHANTA MAHADEVAIAH AND URSULA MITTWOCH \\ Department of Genetics and Biometry, The Galton Laboratory, Wolfson House, \\ 4 Stephenson Way, London NW1 2HE
}

Tertiary trisomic $T s\left(5^{12}\right) 31 \mathrm{H}$ mice are derived from the reciprocal translocation $T(5 ; 12) 31 \mathrm{H}$ and exhibit male sterility. Pachytene chromosomes in spermatocytes and oocytes were analysed in surface-spread, silver-stained preparations in the electron microscope. The material was derived from two tertiary trisomic males and three female embryos. Additional gametocytes from two chromosomally 
normal males and two female embryos born to trisomic mothers were also analysed. In $T s\left(5^{12}\right) 31 H$ males, the small univalent was associated with the $X Y$ bivalent in most cells. In four out of 41 cells of females, the univalent was folded back on itself in what appeared to be a synaptonemal complex. In a small proportion of cells in both sexes, the $5^{12}$ chromosome was synapsed with an autosome. Synaptic errors not affecting the $5^{12}$ chromsome were seen more frequently in females than in males. There was no apparent difference in the incidence of such errors between trisomic and control males. These findings will be discussed in relation to the sterility of tertiary trisomic males.

\title{
The leader cell hypothesis of cytodifferentiation
}

\author{
BY D. J. PRITCHARD \\ Department of Human Genetics, University of Newcastle-upon-Tyne
}

A new theory of cytodifferentiation is proposed, based on observations of transdifferentiating cultures of neural retina. It is suggested that differentiation in a population of competent cells is pioneered by scattered 'leader cells' uniquely responsive to the appropriate inductive factors, which then influence their less responsive neighbours to adopt a similar phenotype. Differentiation of pigment leader cells is promoted by physiological conditions that have no effect on rate of spread of pigmentation to adjacent cells. This suggests that the initiation and secondary spread of pigmentation are brought about by mobilization of somewhat different aspects of cell physiology.

\section{A specific locus experiment with mainly dominant visible results}

\author{
By A. G. SEARLE AND C. V. BEECHEY \\ MRC Radiobiology Unit, Harwell, Oxon OX11 ORD, England
}

The initial object of this experiment was to induce and study deficiencies in the mouse. A tester stock was constructed, homozygous for three pairs of closely linked recessives $(a b p, d s e, r u e p)$; a fourth $(p r u-2)$ is being added. Such females have been mated to wild type males given two doses of $5 \mathrm{~Gy}$ (500 rad) X-rays $24 \mathrm{~h}$ apart, with spermatogonial sampling, since this regime is effective for inducing specific locus mutations, including deletions. However, of over 7000 progeny scored so far only 4 have been mutant at specific loci, namely a cluster of 3 at the $d$ locus and one at $e p$. In contrast with this shortage of recessives has been the detection of 20 dominant visibles, more than would be expected. This has been partly due to a deliberate attempt to find dominants affecting growth by testing mice which were markedly smaller than the rest of the litter at weaning age. In this way 9 heritable small mutations have been detected out of 27 tested. Others died or did not breed but a rough estimate can be made of the total load from such dominants. (Supported by EURATOM contract no. BIO/E/452/81/UK.) 


\title{
Nondisjunction estimates for wild Robertsonian heterozygote common shrews (Sorex araneus L.)
}

\author{
BY J. B. SEARLE \\ Department of Agricultural Science, University of Oxford
}

Among mammals, estimates of anaphase I nondisjunction in heterozygotes for Robertsonian chromosomal rearrangements have been made from studies of captive-bred individuals usually with a genetic background which derives partly or completely from laboratory stock. These estimates may show little relationship to nondisjunction frequencies in Robertsonian heterozygotes from natural polymorphic populations and thus it may not be valid to use them for evolutionary inference. I have estimated anaphase I nondisjunction in Robertsonian heterozygote common shrews from natural polymorphic populations by metaphase II counts in wild-caught adult males and by karyotypic analysis of foetuses in wild-caught adult females. For animals collected from the Oxford area, where the Robertsonian polymorphism involves 5 arm combinations, nondisjunction estimates range from 0.8 to $2.5 \%$ per heterozygous arm combination and 1.2 to $3.7 \%$ per heterozygous individual (some individuals tested were multiple heterozygotes). These data indicate that nondisjunction rates in wild Robertsonian heterozygote common shrews are low.

\section{Male sexual differentiation in mice lacking $\mathrm{H}-\mathrm{Y}$ antigen}

\author{
By ELIZABETH SIMPSON AND ANNE MCLAREN* \\ Transplantation Biology Section, Clinical Research Centre, Watford Road, \\ Harrow, Middlesex HA1 3 UJ \\ * MRC Mammalian Development Unit, University College London, Wolfson \\ House, 4 Stephenson Way, London NW1 $2 H E$
}

We have detected a variant $S x r$ fragment which appears no longer to code for $\mathrm{H}-\mathrm{Y}$ histocompatibility antigen. The implications of this will be discussed.

\section{Chromosomal maps from linkage and physical data}

\section{By PATRICIA SIMPSON}

\section{AFRC Animal Breeding Research Organisation, Edinburgh}

Linkage plays an important role in modern genetics. In man it is used to detect disease associations and in genetic counselling; in domestic animals it may form the basis of selection and in laboratory animals it can be used to identify gene products responsible for genetic traits. Physical data and recombination data can 
be used to form two quite distinct chromosomal maps. Distances between loci can be found by counting recombinant and non-recombinant events in informative families, and combined to form a linkage map with distances measured in centimorgans. Physical data are needed to assign linkage groups to specific chromosomes. These data are obtained from heterochromatic bands, in situ hybridization, inversions, deletions and other chromosomal rearrangements and give rise to a physical map. The linkage map and the physical map are measured on different scales but the chiasmata frequency, or observed rate of crossing over, can be used to combine these two maps provided we assume that the chiasmata are the recombination sites. Chiasmata frequencies have been found for only a small number of animals, principally Drosophila and man, but these are qualitatively very similar. Data on human chromosome 2 were used to find a chromosomal map. Linkage groups were established which could not be established from the physical or linkage data alone. Molecular genetics is a rapidly developing field and these methods will increase in importance as interest in in situ hybridization and RFLP linkage increases.

Based on work done at PGL, University of Hawaii at Manoa.

\section{Structural and functional correlates in hereditary hearing imr airment}

\section{BY KAREN P. STEEL AND GREGORY R. BOCK \\ MRC Institute of Hearing Research, University Park, Nottingham NG7 2RD}

Hereditary hearing impairment may be classified into four groups according to the nature of the lesion. In one group, the abnormality occurs in the central auditory pathways, and in the second group, the morphogenetic processes are affected leading to gross structural deformities of the labyrinth. The last two groups show a normal structural appearance of the bony labyrinth, but there are developmental abnormalities within the cochlea. One of these groups is the neuroepithelial group, in which the earliest detected anomaly occurs in the neuroepithelium of the organ of Corti, and the deafness mouse mutant is an example of this. Hair cells fail to differentiate normally and eventually degenerate, showing no signs of response to sound stimuli at any time during development. The stria vascularis, on the other hand, has a normal appearance and produces an endocochlear potential (E.p.; the d.c. resting potential in the scala media) of normal magnitude. The final group shows cochleo-saccular defects. Our recent work on one example of the cochleo-saccular group, viable dominant spotting, shows that the main lesion occurs in the stria vascularis, which fails to produce an E.P in most of the mutants examined. Many hair cells are present and normal in appearance, and show responses to sound although only at high intensities. The hair cell degeneration that is seen, and the raised thresholds to sound, may be secondary effects of the lack of E.P. 


\title{
More observations on the distribution of melanocytes in aggregation Sash $\leftrightarrow$ wild-type chimaeras
}

\author{
By D. A. STEPHENSON AND P. H. GLENISTER \\ MRC Radiobiology Unit, Harwell, Didcot, Oxon OX11 ORD, U.K.
}

Several loci in the mouse control the distribution of melanocytes throughout the body. Although the mode of action of some spotting loci have been investigated experimentally the conclusions are not in total agreement with those deduced from in vivo studies. Observations on the distribution of melanocytes in $W^{s h} / W^{s h} \leftrightarrow$ $f z l n / f z l n b g b g$ aggregation chimaeras suggested that the presence of the $W^{s h}$ gene modifies the behaviour of the non- $W^{\text {sh }}$ melanocytes to produce a phenotype similar to the $W^{s h}$ heterozygote (Stephenson, Genet. Res. 41, 319, 1983). To determine whether $W^{s h}$ gene dosage modulates the extent and distribution of the melanocyte population, aa $W^{s h}+G p i-1 s^{a} G p i-1 s^{a} \leftrightarrow G p i-1 s^{b} r u-2 p / G p i-1 s^{b} r u-2 p$ aggregation chimaeras were constructed. Two out of three chimaeras displayed a non-pigmented band and patches of full pigmentation in the region of the body where the $W^{s h}$ band occurs in $W^{s h}$ heterozygotes. The presence of fully pigmented patches in the coat excludes the claim that melanocytes bearing $W$ genes are at a competitive disadvantage (Gordon, Differentiation 9, 19, 1977 ; Mayer, J. Expt. Zool. 210, 81, 1979). The other chimaera displayed no evidence of the $W^{s h}$ component in the coat but showed patches of wild-type pigmentation in both eyes. These and other preliminary observations on the mosaicism in other tissues will be presented.

\section{Rates of occurrence of Peruvian mouse mutants}

\author{
BY MARGARE'T E. WALLACE \\ Department of Genetics, University of Cambridge, U.K.
}

The laboratory-bred population of mice from the third trapping in Peru (Peru-Coppock: Mouse Newsletter 67, 12, 1982) has reached its 16th generation of close inbreeding. Current descendants trace to 100 of the trapped mice. The early generations should throw up recessives originating in the population before trapping, plus recessives newly arisen within the laboratory stock. Later generations should produce only newly arisen recessives. Dominant mutants have arisen newly in the matings first producing them. Data showing in which generation each of the Peru mutants was seen, give a basis for dividing them tentatively according to the two origins. Rough estimates of the rate of production of recessives of mixed origin, and of newly arisen mutants (dominant and recessive) from sibmatings are: 1 in 1200 and 1 in 7200 young respectively. Comparison with the Cambridge rate for laboratory mutants is complicated by the varied breeding systems. Comparison with the Jackson (Genetics 57, 319-330, 1967) shows that most of the types of 
mutant seen there are not represented in the Perus, probably because the Perus are unselected in terms of the viability and penetrance. Colour loci may be comparable: the Jackson newly arisen rate is 1 in 500000 and the Peru 1 in 18-19000. Comparison of mutant rates to loci new to science per locus per gamete shows the Peru mice to have 90 times the Jackson rate. A specific locus test for this rate in the Perus would avoid many of the complications and provide for comparison with artificial mutagens such as ENU. 